\title{
Classification of total nasal obstruction in 10,033 cases by 4-phase -rhinomanometry
}

\author{
Klaus Vogt ', Klaus-Dieter Wernecke' ${ }^{2}$, Mara Argale', Krista Kaulina' \\ ${ }^{1}$ University of Latvia, Faculty of Medicine, Center of Experimental Medicine, Riga, Latvia \\ ${ }^{2}$ Sostana GmbH, Berlin, Germany
}

\begin{abstract}
BACKGROUND. The measurement of the total nasal resistance by rhinomanometry is of special interest in the functional diagnosis of the nasopharynx and retropalatal space. It can be measured by posterior rhinomanometry or estimated by calculating the total resistance from measurements of both sides. Because the standard parameters of the classic rhinomanometry have to be considered as obsolete, Vertex Resistance and Effective Resistance and their logarithmic derivations have been introduced with 4-phase-rhinomanometry.

MATERIAL AND METHODS. In the first part, posterior and anterior rhinomanometry have been compared in a study of 32 volunteers. The disadvantages of posterior rhinomanometry have been apparent because of inconsistent statistic results. To compare both methods is useful in cooperative patients for the diagnosis of postnasal pathological alterations. By analyzing the rhinomanometric databases of 5 hospitals in 10,033 untreated patients and in 8,246 patients after decongestion, the total nasal resistance could be determined.

RESULTS. Because only the logarithmic values for effective and vertex resistances are correlated with the sensing of obstruction, these values have been used to classify the total obstruction in 5 classes. The descriptive data of every class are given. The here-described classes are valid for Caucasian noses. The calculated data can be delivered on request by the authors.

CONCLUSION. The here-presented parameters for measuring the resistance of the nasal airway and the classification of the obstruction from a statistically representative material can be recommended for the daily practice in rhinology and rhinosurgery. KEYWORDS: rhinomanometry, 4-phase-rhinomanometry, parameters, total nasal resistance, nasal obstruction
\end{abstract}

\section{INTRODUCTION}

For the determination of the nasal airway resistance as a diagnostic method - in particular before functional nasal surgery - active anterior rhinomanometry with separate measurements of both sides is the method of first choice, because the surgeon is interested in detailed information about the surgical field. However, the determination of total nasal airway resistance by rhinomanometry gets an increasing interest within the clinical rhinology as well as in the framework of sleep medicine and for the evaluation of drugs effective to improve nasal obstruction. In children, the determination of the total nasal resistance is used for the evaluation of the nasal obstruction due to adenoid hypertrophy ${ }^{1}$. It is possible to perform nasal provoca- tion tests as unilateral tests, as well as by measuring the total nasal resistance. Furthermore, it is possible by subsequent anterior and posterior rhinomanometry to determine the partial resistance of the nasopharyngeal and retropharyngeal space. The information about the total nasal airway resistance is also of interest, if asymmetric breathing through the nose is not the primary target, but the possible impairment of nasal breathing within the function of the entire upper airway.

In a recent paper, Merkle et al. ${ }^{2}$ published a review of reference intervals after averaging the results of 62 studies from 1979 to 2013 in different races for the "normal" noses. As a conclusion, it was stated that it would be desirable to have statistically representative data as a basement for the respective comparisons. 
The paper reports meticulously about resistance measurements at a pressure of $150 \mathrm{~Pa}, 100 \mathrm{~Pa}$ or $75 \mathrm{~Pa}$. Finally, reference intervals for these parameters are given. The investigated parameters are 1-point-measurements, by which estimations of the energetic of the nasal airway are deducted, which are not anymore acceptable by physical and mathematical reasons.

Under the auspices of quality management of diagnostic in rhinology, such estimations have to be replaced by reliable measured parameters ${ }^{3,4}$. The basic principles of 4- phase-rhinomanometry are not only the resolution of the nasal breath in an ascending and descending inspiratory and expiratory part, but also the introduction of correct parameters with high diagnostic power. Such parameters are Effective Resistance (Reff) and Vertex Resistance (VR). These parameters, after logarithmic transformation (LReff, LVR), are statistically correlated with the subjective feeling of obstruction as obtained by a VAS-scale". The term "effective resistance" was deducted from electro engineering and means the integrated resistance measured about the entire length of a nasal breath or its inspiratory or expiratory part. "Vertex Resistance" is measured at the top of the breathing wave, where the timedependent curves for the narino-choanal pressure difference and for the nasal flow are parallel. This point is the only one of the breathing cycle, where the calculation of a resistance as a linear quotient of pressure and flow is correct.

In previous papers ${ }^{3,4}$, the derivation of those parameters and the errors of the "classic" rhinomanometry are repeatedly described, as well as the results of a retrospective multicentric analysis of 36,563 unilateral measurements by 4-phase-rhinomanometry with a following clinical classification of obstruction. This analysis is now followed by the evaluation of the statistical results and classification of representative data for the total nasal resistance.

Numeric information of the total nasal airway resistance can be obtained in 2 different ways:

1. Active posterior rhinomanometry. The difference to the active anterior rhinomanometry is given by the intraoral fixation of the pressure measurement tube by the lips for measuring the pressure difference between the nasopharynx and the mask. These measurements include also the resistance of the space between choanae and the lower end of the soft palate, i.e. the nasopharynx. The obtained numerical results represent measurements. Erroneous or non-evaluable results can occur if the patient is not able to keep the tongue relaxed during the data uptake or the process is interrupted by gagging or swallowing.

2. Calculation of the total nasal resistance from the resistances of both nasal sides measured by active anterior rhinomanometry using the formula of parallel electric resistors (Kirchhoff): The numeric results after this mathematical transformation are estimations out of 2 measurements. The diagnostic value can be considered very high if the calculated resistances are obtained by measurements of the Effective Resistance (Reff) or Vertex Resistance (VR) and not by the incorrect linear resistances calculated as quotient of 150 , 100 or $75 \mathrm{~Pa}$ by the measured flow (see below!).

If both measurements can be correctly performed in the same patient, it is principally possible to estimate the partial resistance of the nasopharynx in cases where this area is narrowed by pathological formations as tumors, adenoids or scars.

The aim of this paper was to investigate the differences in measurement results for the total nasal resistance obtained by posterior and anterior rhinomanometry and to present a clinical classification of the total nasal resistance in a clinical representative material. Following our retrospective analysis of 20,069 measurements without treatment and 16,494 measurements after decongestion by xylometazoline for the active anterior rhinomanometry in unilateral measurements by 4 -phase-rhinomanometry (4PR), the calculated classified resistances for the total nasal resistance shall be presented with correct parameters, which are correlated to the subjective sensation of nasal obstruction.

Because of the methodical differences to determine the total nasal resistance, it was necessary to compare these methods before in a minor preparatory study.

\section{MATERIAL AND METHODS}

All measurements have been carried out by using the 4PR- rhinomanometer models HRR3 or 4RHINO (Rhinolab GmbH, Germany) with software 3.57, 4.31 or 5.01. The software of this system is WINDOWS based and the format of the data stored in the databases is identical since 1999. The following details are important for providing exact measurements and reproducible results:

1. The calibration of the device was controlled in predetermined distances; the calibration of all instruments was correct before the beginning and after the end of the studies.

2. For the coupling of the pressure tube to the nose in active anterior rhinomanometry (AAR), the "tape method" has been applied exclusively. The use of any prefabricated coupling element is forbidden in the participating departments. The elastic tape Microfoam (3M) was used. In the introductory study for the measurement of the intraoral differential pressure, a 4x6 $\mathrm{mm}$ silicone tube was used. 
3. Anesthesiological masks of different sizes (Ambu GmbH, Germany) have been chosen. The extranasal "dead space" of the system does not exceed 0,151 including connection pieces and filter housing.

4. All measurements have been carried out after adaptation of the patient to room temperature, in rest and in upright sitting position.

The measurement results are stored as an average of $3-5$ breathing cycles with 2000 data for flow and differential pressure according to the recommendations of the ISOANA 1984. The averaging procedure by splinting was described by Wernecke and $\operatorname{Vogt}^{3}$. By an export function of the 4PR-program results can be directly transferred to text-files for further processing with statistic standard programs. SPSS 22 and Excel 2010 with XL-Stat have been used for statistical evaluation.

The parameters evaluated in both parts of the study are presented in the following table (Table 1).

The definition and derivation of all parameters is extensively described in our previous publications ${ }^{3,4}$. For the calculation of the total resistance from the resistances of both nasal sides measured by AAR, the formula for parallel resistors above was applied.

Part 1: Evaluation of differences between posterior and anterior rhinomanometry for the determination of the total nasal resistance

Material and methods: In 32 volunteers (17 male, 15 female, age $20-74$ years) without complaints or clinical signs of an obstructive nasal disease, an active anterior rhinomanometry (AAR) was carried out, followed by posterior rhinomanometry (APR). The measurements have been repeated as decongestion test 10 minutes after application of $0.1 \%$ xylometazoline spray. 2 patients had to be excluded from the study because they have not been able to keep the tongue relaxed for APR.

Results: In Table 2 and Figure 1 the results for the resistance values of "classic rhinomanometry" are listed. As expected, clear differences can be seen between the 4 breathing phases, corresponding to the previous results of measurements of one side. These values are noted here only to allow comparisons with references ${ }^{2}$ and to point out the importance of the 4 phases of the breathing cycle.

It is visible that the resistances in phase 2 and 3 are significantly higher than in phase 1 and 4 , which is more apparent in untreated noses. The number of valid measurements was reduced because some volunteers did not reach the pressure level of $150 \mathrm{~Pa}$. It is clearly visible that the measured resistances by APR are statistically higher than the resistances by AAR. That can be underlined by considering the flow sum measured either in both sides or by APR (Figure 2): the measured total nasal airflow is clearly lower than the flow sum of both sides by unilateral measurements.

The results for the 4PR specific parameters are summarized in Table 3 and visualized in Figure 3.

As a main result, there are only marginal differences between VRin, VRex, Reffin, Reffex and Reff, but the values in APR are significantly higher in APR before decongestion. Therefore, it is of much higher

Table 1

Definition of Resistance values in 4-Phase-Rhinomanometry

\begin{tabular}{|c|c|c|}
\hline & Value & $\begin{array}{c}\text { Logarithmic transformation } \\
\log \left(10^{*} \text { value }\right)\end{array}$ \\
\hline Linear Resistance in $150 \mathrm{~Pa}$, phase 1 & R1 & LR1 \\
\hline Linear Resistance in $150 \mathrm{~Pa}$, phase 2 & R2 & LR2 \\
\hline Linear Resistance in $150 \mathrm{~Pa}$, phase 3 & R3 & LR3 \\
\hline Linear Resistance in $150 \mathrm{~Pa}$, phase 4 & R4 & LR4 \\
\hline Vertex Resistance in inspiration & VRin & LVRin \\
\hline Vertex Resistance in expiration & VRex & LVRex \\
\hline Effective Resistance, inspiration & Reffin & LReffin \\
\hline Effective Resistance, expiration & Reffex & LReffex \\
\hline Effective Resistance, total breath & Reff & LReff \\
\hline
\end{tabular}


Table 2

Total resistances in 150Pa in 4 breathing phases

\begin{tabular}{|c|c|c|c|c|c|c|c|c|}
\hline & Phase 1 & Phase 2 & Phase 3 & Phase 4 & Phase 1 & Phase 2 & Phase 3 & Phase 4 \\
\hline Mean & 0,523 & 0,713 & 0,722 & 0,569 & 0,977 & 1,156 & 0,895 & 0,698 \\
\hline Median & 0,400 & 0,414 & 0,481 & 0,457 & 0,528 & 0,532 & 0,552 & 0,526 \\
\hline SD & 0,363 & 0,624 & 0,495 & 0,386 & 1,202 & 1,343 & 1,514 & 1,300 \\
\hline $\mathrm{N}$ & 29 & 29 & 25 & 25 & 25 & 25 & 22 & 22 \\
\hline \multicolumn{9}{|c|}{ after decongestion } \\
\hline & Phase 1 & Phase 2 & Phase 3 & Phase 4 & Phase 1 & Phase 2 & Phase 3 & Phase 4 \\
\hline Mean & 0,352 & 0,392 & 0,404 & 0,349 & 0,874 & 1,081 & 0,709 & 0,616 \\
\hline Median & 0,318 & 0,335 & 0,339 & 0,332 & 0,394 & 0,441 & 0,380 & 0,373 \\
\hline SD & 0,160 & 0,207 & 0,201 & 0,153 & 1,329 & 1,813 & 1,192 & 0,937 \\
\hline $\mathrm{N}$ & 29 & 29 & 29 & 29 & 23 & 23 & 19 & 19 \\
\hline
\end{tabular}

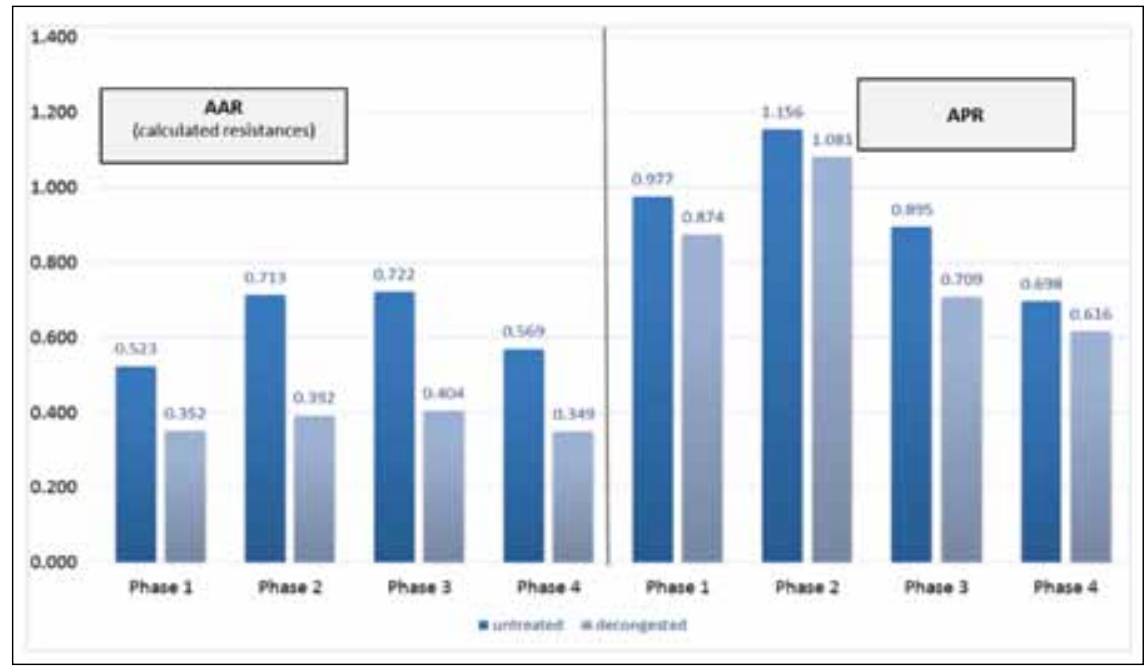

Figure 1 The results for the resistance values of "classic rhinomanometry"

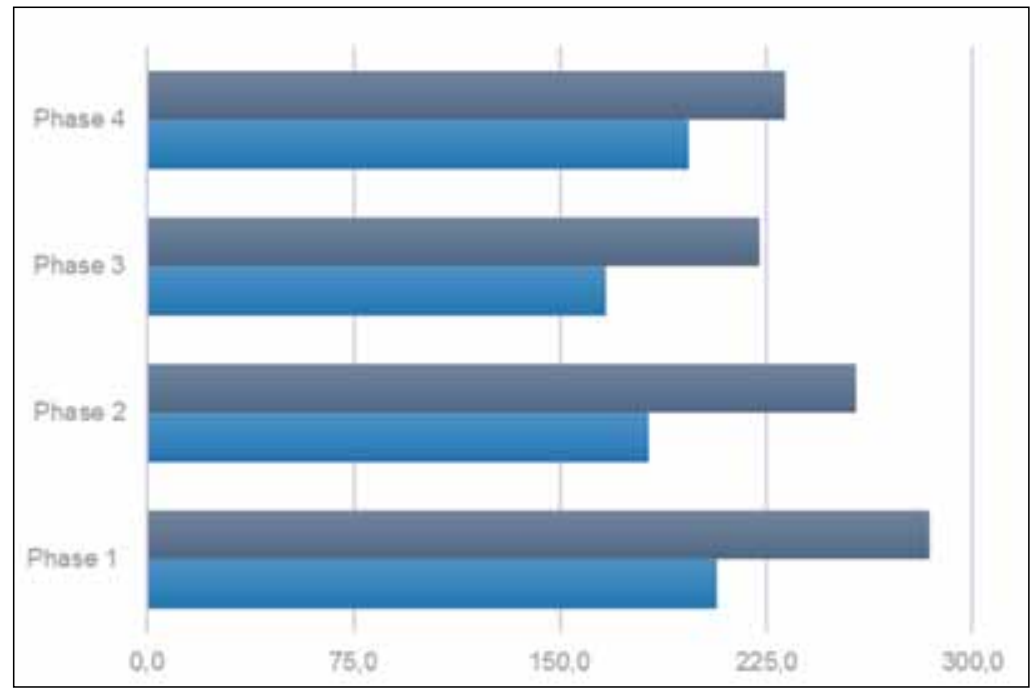

Figure 2 Statistical differences in total nasal flow measured by AAR and APR 
Table 3

The results for the 4PR specific parameters

\begin{tabular}{|c|c|c|c|c|c|c|c|c|c|c|}
\hline \multirow[b]{3}{*}{ untreated } & \multicolumn{5}{|c|}{ AAA, calculated resistances } & \multicolumn{5}{|c|}{ APR, measured resistances } \\
\hline & \multicolumn{10}{|c|}{ Values non transformed, $\mathrm{N}=\mathbf{3 0}$} \\
\hline & Vrin & Vrex & Reffin & Reffex & Reff & Vrin & Vrex & Reffin & Reffex & Reff \\
\hline Mean & 0,798 & 0,719 & 0,779 & 0,687 & 0,746 & 1,203 & 0,942 & 1,187 & 0,977 & 1,118 \\
\hline Median & 0,542 & 0,479 & 0,496 & 0,451 & 0,504 & 0,619 & 0,578 & 0,616 & 0,635 & 0,655 \\
\hline SD & 0,575 & 0,526 & 0,580 & 0,501 & 0,543 & 1,534 & 1,105 & 1,513 & 1,136 & 1,339 \\
\hline \multicolumn{11}{|c|}{ After decongestion } \\
\hline Mean & 0,549 & 0,494 & 0,554 & 0,514 & 0,542 & 0,880 & 0,615 & 0,861 & 0,647 & 0,780 \\
\hline Median & 0,443 & 0,452 & 0,444 & 0,438 & 0,446 & 0,439 & 0,386 & 0,421 & 0,362 & 0,417 \\
\hline \multirow[t]{2}{*}{ SD } & 0,385 & 0,225 & 0,416 & 0,369 & 0,398 & 1,344 & 0,959 & 1,415 & 0,949 & 1,193 \\
\hline & \multicolumn{10}{|c|}{ Values after logarithmic transformation, $\mathrm{N}=30$} \\
\hline untreated & LVRin & LVRex & Lreffin & Lreffex & Lreff & LVRin & LVRex & Lreffin & Lreffex & Lreff \\
\hline Mean & 0,794 & 0,746 & 0,777 & 0,724 & 0,762 & 0,835 & 0,759 & 0,834 & 0,761 & 0,824 \\
\hline Median & 0,734 & 0,679 & 0,695 & 0,653 & 0,702 & 0,792 & 0,762 & 0,789 & 0,803 & 0,816 \\
\hline SD & 0,312 & 0,317 & 0,320 & 0,320 & 0,317 & 0,459 & 0,455 & 0,451 & 0,471 & 0,445 \\
\hline \multicolumn{11}{|c|}{ After decongestion } \\
\hline Mean & 0,656 & 0,652 & 0,662 & 0,630 & 0,653 & 0,730 & 0,596 & 0,706 & 0,608 & 0,687 \\
\hline Median & 0,646 & 0,655 & 0,648 & 0,641 & 0,649 & 0,643 & 0,586 & 0,624 & 0,558 & 0,620 \\
\hline SD & 0,267 & 0,195 & 0,255 & 0,260 & 0,255 & 0,384 & 0,353 & 0,392 & 0,375 & 0,377 \\
\hline
\end{tabular}

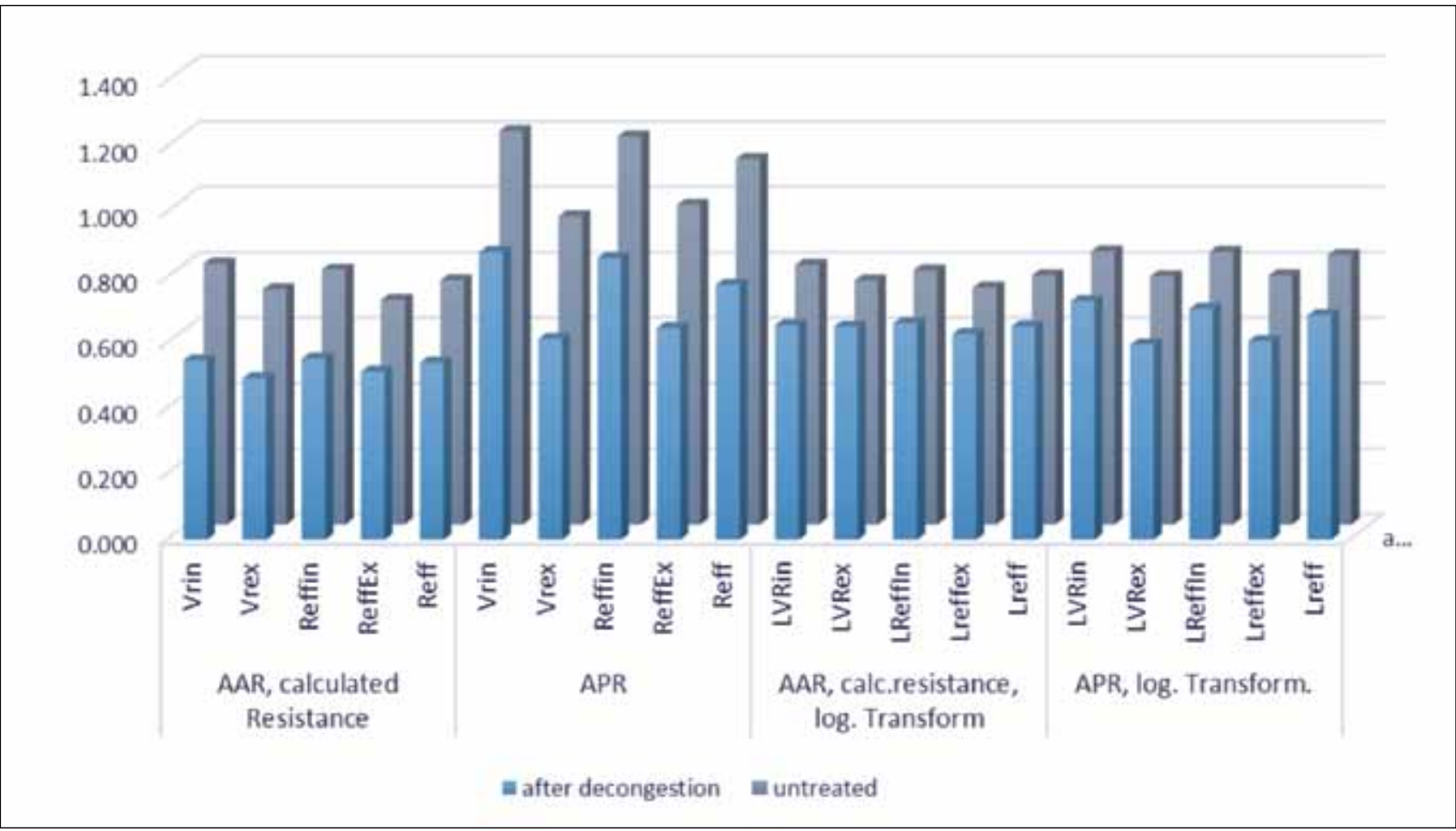

Figure 3 The results for the 4PR specific parameters 
clinical interest to see the intraindividual differences for the total nasal resistance depending on the measurement methods. Figure 4 clearly shows such differences, which may appear in patients or volunteers even without pathological symptoms or findings.

Part 2: Clinical classification of obstruction by determination of total nasal resistance

Material and methods: The rhinomanometric databases of 5 different German ENT-hospitals ${ }^{1-5}$ using 4-phase-rhinomanometry for more than 5 years have been analyzed in this study. 3 departments deal with general otorhinolaryngology, 2 hospitals deal with facial-plastic surgery. The range of age was $14-82$ years. In 20,069 untreated nasal sides, active anterior rhinomanometry was carried out. 16,494 measurements were subsequently followed by a decongestion test with xylo- metazoline $0.1 \%$ spray and a second measurement 10 minutes later. The total nasal resistance could be calculated in 10,033 of these patients, furthermore in 8,246 patients after decongestion. The results have been classified by $20 \%$ percentiles. The main statistical results for the single classes and for the entire cohort obtained from the measured values as well as after logarithmic transformation of the 4PR-specific values are given in Table 4 . The analyzed logarithmic values have been calculated as decadic logarithms of the 10-fold measured value to avoid negative logarithmic results for better handling in clinical routine ${ }^{2,3}$. Example: LVRin $=\log$ $\left(10^{*}\right.$ VRin $)$. Because the resistances at $150 \mathrm{~Pa}$ are noted in this paper only for the comparison with references, they have not been logarithmically transformed.

Results: The numeric results of the statistical evaluation are summarized in Table 4.

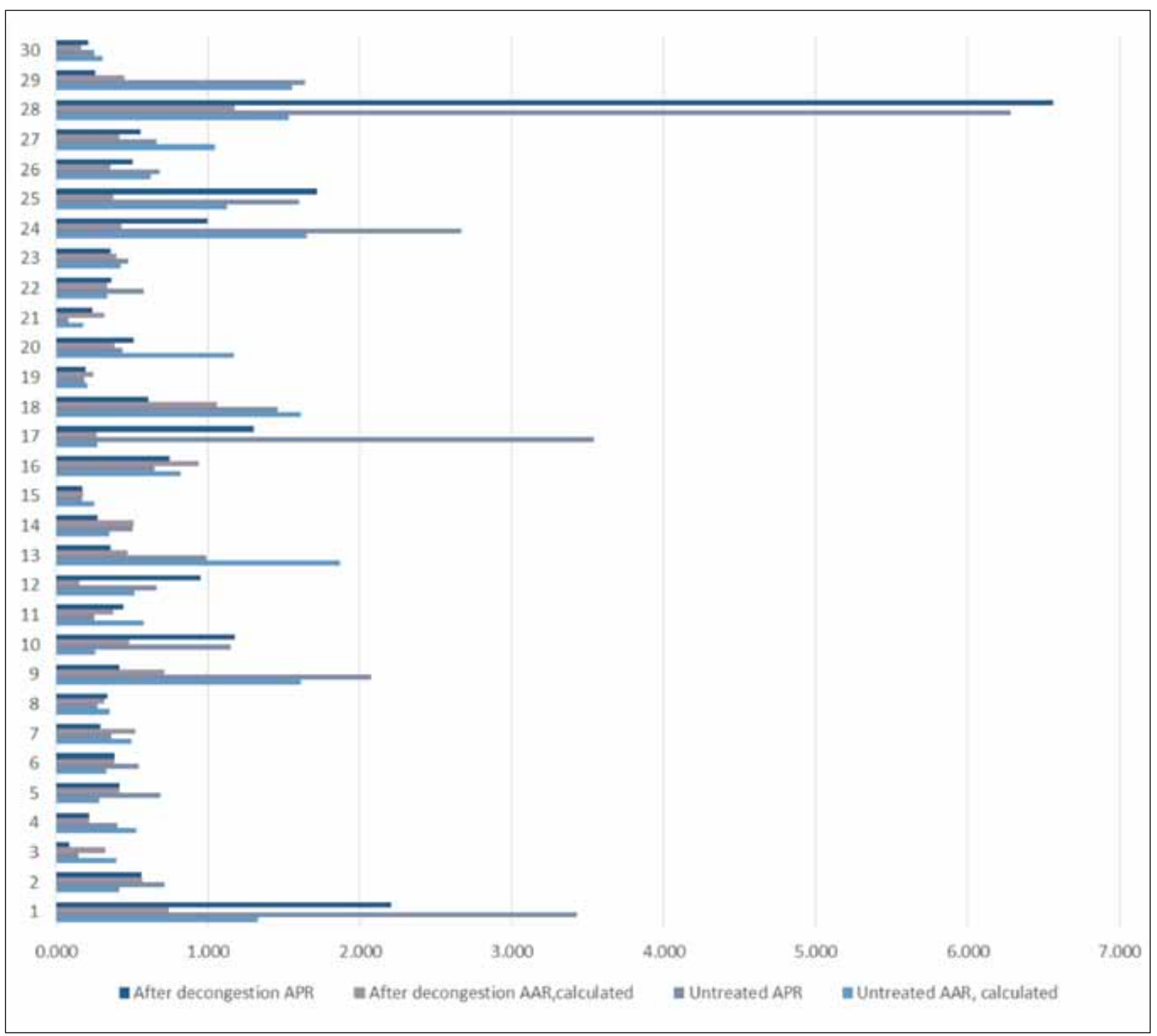

Figure 4 Intraindividual differences for the total nasal resistance depending on the measurement methods 
Table 4

Descriptive statistics for classified parameters in total nasal resistance

\begin{tabular}{|c|c|c|c|c|c|c|c|c|c|c|c|c|c|}
\hline & \multirow[b]{2}{*}{ Class } & \multicolumn{6}{|c|}{ Untreated } & \multicolumn{6}{|c|}{ After decongestion } \\
\hline & & 1 & 2 & 3 & 4 & 5 & Unclassified & 1 & 2 & 3 & 4 & 5 & Undassified \\
\hline R1 & range & $<=0.195$ & $0.196-0.260$ & $0.261-0.335$ & $0.336-0.530$ & $0.531+$ & & $<=0.171$ & $0.172-0.224$ & $0.225-0.292$ & $0.293-0.413$ & $0.414+$ & \\
\hline $\mathrm{Rin} 150 \mathrm{~Pa}$ & mean & 0.165 & 0.228 & 0.295 & 0.414 & 0.951 & 0.42 & 0.151 & 0.197 & 0.255 & 0.343 & 0.684 & 0.318 \\
\hline \multirow[t]{2}{*}{ phase 1} & SD & 0.023 & 0.019 & 0.021 & 0.055 & 0.574 & 0.371 & 0.170 & 0.015 & 0.019 & 0.034 & 0.451 & 0.259 \\
\hline & $\mathrm{N}$ & 1191 & 1954 & 1886 & 2366 & 1752 & 9149 & 1080 & 1910 & 1751 & 1662 & 1335 & 7738 \\
\hline R2 & range & $<=0.205$ & $0.206-0.287$ & $0.288-0.392$ & $0.393-0.620$ & $0.621+$ & & $<=0.177$ & $0.178-0.244$ & $0.254-0.317$ & $0.318-0.507$ & $0.508+$ & \\
\hline R in $150 \mathrm{~Pa}$ & mean & 0.171 & 0.246 & 0.335 & 0.487 & 1.415 & 0.561 & 0.153 & 0.209 & 0.278 & 0.393 & 1.051 & 0.404 \\
\hline \multirow[t]{2}{*}{ phase 2} & SD & 0.026 & 0.024 & 0.029 & 0.064 & 1.362 & 0.785 & 0.019 & 0.019 & 0.021 & 0.054 & 0.866 & 0.474 \\
\hline & $\mathrm{N}$ & 1178 & 1954 & 1981 & 1953 & 1960 & 9026 & 1000 & 2010 & 1489 & 1823 & 1328 & 7650 \\
\hline R3 & range & $<=0.222$ & $0.223-0.300$ & $0.301-0.434$ & $0.435-0.694$ & $0.695+$ & & $<=0.198$ & $0.199-0.266$ & $0.267-0.343$ & $0.344-0.545$ & $0.546+$ & \\
\hline $\mathrm{R}$ in $150 \mathrm{~Pa}$ & mean & 0.075 & 0.261 & 0.362 & 0.539 & 1.332 & 0.537 & 0.17 & 0.232 & 0.302 & 0.424 & 0.965 & 0.41 \\
\hline \multirow[t]{2}{*}{ phase 3} & SD & 0.754 & 0.023 & 0.038 & 0.072 & 1.021 & 0.669 & 0.023 & 0.019 & 0.022 & 0.058 & 0.734 & 0.406 \\
\hline & $\mathrm{N}$ & 1069 & 1628 & 2291 & 1943 & 1680 & 8644 & 1033 & 1779 & 1418 & 1777 & 1252 & 7259 \\
\hline R4 & range & $<=0.189$ & $0.190-0.261$ & $0.262-0.334$ & $0.335-0.525$ & $0.526+$ & & $<=0.168$ & $0.169-0.216$ & $0.217-0.280$ & $0.281-0.399$ & $0.400+$ & \\
\hline Rin $150 \mathrm{~Pa}$ & mean & 0.068 & 0.225 & 0.295 & 0.412 & 1.017 & 0.41 & 0.147 & 0.191 & 0.246 & 0.329 & 0.655 & 0.308 \\
\hline \multirow{2}{*}{ phase 4} & SD & 1.010 & 0.02 & 0.021 & 0.054 & 0.964 & 0.623 & 0.018 & 0.013 & 0.019 & 0.034 & 0.372 & 0.239 \\
\hline & $\mathrm{N}$ & 1076 & 2034 & 1772 & 2170 & 1559 & 8611 & 1039 & 1664 & 1702 & 1568 & 1254 & 7227 \\
\hline \multirow[t]{4}{*}{ VR, inspiration } & range & $<=0.282$ & $0.283-0.400$ & $0.401-0.549$ & $0.550-0.851$ & $0.852+$ & & $<=0.246$ & $0.247-0.324$ & $0.325-0.429$ & $0.430-0.631$ & $0.632+$ & \\
\hline & mean & 0.203 & 0.342 & 0.468 & 0.676 & 1.556 & 0.649 & 0.193 & 0.284 & 0.372 & 0.517 & 1.098 & 0.493 \\
\hline & SD & 0.06 & 0.034 & 0.042 & 0.086 & 1.250 & 0.738 & 0.042 & 0.022 & 0.031 & 0.056 & 0.737 & 0.461 \\
\hline & $\mathrm{N}$ & 2007 & 2007 & 2006 & 2006 & 2007 & 10033 & 1650 & 1649 & 1649 & 1649 & 1649 & 8246 \\
\hline \multirow[t]{4}{*}{ VR, expiration } & range & $<=0.268$ & $0.269-0.371$ & $0.372-0.502$ & $0.503-0.744$ & $0.745+$ & & $<=0.235$ & $0.236-0.298$ & $0.299-0.382$ & $0.383-0.532$ & $0.533+$ & \\
\hline & mean & 0.191 & 0.319 & 0.432 & 0.603 & 1.331 & 0.575 & 0.184 & 0.267 & 0.337 & 0.448 & 0.854 & 0.417 \\
\hline & SD & 0.057 & 0.029 & 0.037 & 0.069 & 0.858 & 0.557 & 0.043 & 0.018 & 0.025 & 0.042 & 0.447 & 0.309 \\
\hline & $\mathrm{N}$ & 2006 & 2008 & 2005 & 2008 & 2006 & 10033 & 1649 & 1649 & 1649 & 1649 & 1650 & 8246 \\
\hline \multirow[t]{4}{*}{ LogVR, inspir. } & range & $<=0.450$ & $0.451-0.603$ & $0.604-0.740$ & $0.741-0.930$ & $0.931+$ & & $<=0.391$ & $0.392-0.510$ & $0.511-0.633$ & $0.634-0.800$ & $0.801+$ & \\
\hline & mean & 0.28 & 0.532 & 0.669 & 0.826 & 1.137 & 0.689 & 0.273 & 0.452 & 0.569 & 0.711 & 0.994 & 0.6 \\
\hline & SD & 0.17 & 0.044 & 0.039 & 0.055 & 0.189 & 0.311 & 0.119 & 0.034 & 0.036 & 0.047 & 0.178 & 0.264 \\
\hline & $\mathrm{N}$ & 2007 & 2007 & 2006 & 2007 & 2006 & 10033 & 1650 & 1649 & 1649 & 1649 & 1649 & 8246 \\
\hline \multirow[t]{4}{*}{ Log VR, exp. } & range & $<=0.428$ & $0.429-0.570$ & $0.571-0.701$ & $0.702-0.872$ & $0.873+$ & & $<=0.371$ & $0.372-0.474$ & $0.475-0.582$ & $0.583-0.726$ & $0.727+$ & \\
\hline & mean & 0.254 & 0.502 & 0.634 & 0.777 & 1.076 & 0.649 & 0.247 & 0.425 & 0.527 & 0.64 & 0.896 & 0.549 \\
\hline & SD & 0.168 & 0.04 & 0.037 & 0.049 & 0.183 & 0.298 & 0.132 & 0.03 & 0.032 & 0.041 & 0.159 & 0.238 \\
\hline & $\mathrm{N}$ & 2007 & 2006 & 2006 & 2008 & 2006 & 10033 & 1649 & 1649 & 1649 & 1649 & 1650 & 8246 \\
\hline \multirow[t]{4}{*}{ Reff, inspir. } & range & $<=0.269$ & $0.270-0.381$ & $0.382-0.528$ & $0.529-0.840$ & $0.841+$ & & $<=0.234$ & $0.235-0.309$ & $0.310-0.414$ & $0.415-0.623$ & $0.624+$ & \\
\hline & mean & 0.193 & 0.325 & 0.448 & 0.659 & 1.678 & 0.66 & 0.184 & 0.27 & 0.357 & 0.504 & 1.189 & 0.5 \\
\hline & SD & 0.057 & 0.032 & 0.042 & 0.089 & 1.757 & 0.949 & 0.04 & 0.021 & 0.031 & 0.059 & 1.047 & 0.591 \\
\hline & $\mathrm{N}$ & 2007 & 2006 & 2006 & 2008 & 2006 & 10033 & 1648 & 1649 & 1649 & 1651 & 1649 & 8246 \\
\hline \multirow[t]{4}{*}{ Reff, exp. } & range & $<=0.250$ & $0.251-0.346$ & $0.347-0.471$ & $0.472-0.709$ & $0.710+$ & & $<=0.220$ & $0.221-0.280$ & $0.281-0.360$ & $0.361-0.504$ & $0.505+$ & \\
\hline & mean & 0.178 & 0.298 & 0.404 & 0.57 & 1.316 & 0.553 & 0.172 & 0.25 & 0.317 & 0.424 & 0.828 & 0.398 \\
\hline & SD & 0.053 & 0.027 & 0.035 & 0.067 & 0.969 & 0.593 & 0.04 & 0.017 & 0.024 & 0.041 & 0.458 & 0.309 \\
\hline & $\mathrm{N}$ & 2007 & 2007 & 2006 & 2007 & 2006 & 10033 & 1650 & 1649 & 1649 & 1648 & 1650 & 8246 \\
\hline Reff & range & $<=0.265$ & $0.266-0.373$ & $0.374-0.505$ & $0.506-0.787$ & $0.788+$ & & $<=0.230$ & $0.231-0.299$ & $0.300-0.391$ & $0.392-0.569$ & $0.570+$ & \\
\hline & mean & 0.194 & 0.319 & 0.434 & 0.625 & 1.486 & 0.611 & 0.183 & 0.264 & 0.34 & 0.466 & 1.006 & 0.451 \\
\hline & SD & 0.054 & 0.03 & 0.038 & 0.081 & 1.200 & 0.708 & 0.039 & 0.02 & 0.026 & 0.06 & 0.728 & 0.438 \\
\hline & $\mathrm{N}$ & 2006 & 2008 & 2007 & 2006 & 2006 & 10033 & 1649 & 1650 & 1649 & 1649 & 1649 & 8246 \\
\hline Log Reff, inspir. & range & $<=0.429$ & $0.430-0.581$ & $0.582-0.723$ & $0.724-0.924$ & $0.923+$ & & $<=0.369$ & $0.370-0.489$ & $0.490-0.617$ & $0.618-0.794$ & $0.795+$ & \\
\hline & mean & 0.258 & 0.51 & 0.649 & 0.815 & 1.154 & 0.677 & 0.252 & 0.43 & 0.551 & 0.699 & 1.012 & 0.589 \\
\hline & SD & 0.168 & 0.044 & 0.04 & 0.058 & 0.209 & 0.325 & 0.119 & 0.034 & 0.037 & 0.051 & 0.202 & 0.28 \\
\hline & $\mathrm{N}$ & 2007 & 2007 & 2006 & 2006 & 2007 & 10033 & 1649 & 1649 & 1649 & 1650 & 1649 & 8246 \\
\hline Log Reff, exp. & range & $<=0.398$ & $0.399-0.538$ & $0.539-0.673$ & $0.674-0.851$ & $0.852+$ & & $<=0.342$ & $0.343-0.447$ & $0.448-0.557$ & $0.558-0.703$ & $0.704+$ & \\
\hline & mean & 0.226 & 0.473 & 0.605 & 0.753 & 1.062 & 0.624 & 0.22 & 0.396 & 0.5 & 0.625 & 0.87 & 0.524 \\
\hline & SD & 0.161 & 0.404 & 0.038 & 0.051 & 0.194 & 0.303 & 0.128 & 0.03 & 0.032 & 0.042 & 0.165 & 0.242 \\
\hline & $\mathrm{N}$ & 2008 & 2005 & 2008 & 2005 & 2007 & 10033 & 1650 & 1648 & 1649 & 1649 & 1650 & 8246 \\
\hline Log Reff, & range & $<=0.423$ & $0.424-0.571$ & $0.572-0.703$ & $0.704-0.896$ & $0.897+$ & & $<=0.362$ & $0.363-0.475$ & $0.476-0.592$ & $0.593-0.755$ & $0.756+$ & \\
\hline & mean & 0.265 & 0.503 & 0.635 & 0.792 & 1.113 & 0.662 & 0.249 & 0.42 & 0.531 & 0.666 & 0.952 & 0.564 \\
\hline & SD & 0.151 & 0.042 & 0.038 & 0.056 & 0.196 & 0.307 & 0.115 & 0.032 & 0.034 & 0.047 & 0.184 & 0.251 \\
\hline & $\mathrm{N}$ & 2007 & 2008 & 2005 & 2006 & 2007 & 10033 & 1649 & 1650 & 1649 & 1649 & 1649 & 8246 \\
\hline
\end{tabular}


Typical distributions in measured values and after logarithmic transformation are demonstrated in Figure 5 by histograms for Reff and LReff. Histograms and parameters of all classes, as well as of the non-classified material, show a uniform pattern. While the non-logarithmic values are shifted to the left side, logarithmic values are approximately normally (Gauss) distributed, which has been already found in our previous analysis of unilateral measurements. The distributions of the logarithmic vertex resistances (LVRin, LVRex) and the logarithmic effective resistances (LReffex, LReffex, LReff) are very similar.

It should be mentioned that these differences of distribution - as observed before in unilateral measurements - are present because only logarithmic values are correlated with VAS-scales for the subjective sensing of obstruction following Weber-Fechner's law3. For the total nasal resistance correlations of 4PR-specific parameters with subjective data are not available up to now.

The comparison of the main results of the data analysis of the 4PR-specific parameters show the following results as of practical importance:

1. Vertex resistance (VR) is statistically $5.8 \%$ higher in inspiration than in expiration

2. Effective Resistance is $6.4 \%$ higher in inspiration than in expiration. Reff as measured over the en- tire breath is obviously preferably determined by the inspiration: ReffIn $>$ Reff $>$ ReffEx

3 . The total resistance after decongestion is lower, but the differences between the parameters show the same tendency.

That involves for the clinical classification of nasal obstruction an acceptable approximation to use the classification for LReff as well for ReffIn, LReffEx, VRin and VRex. The effective resistance (Reff) and the logarithmic effective resistance (LReff) are the most important parameters, because they are measured parameters representing the integrated nasal resistance within an entire average breath. The remaining parameters can be considered as supplementary diagnostic information, if the curve parts in different breathing phases are diverging (loops, "valve phenomena") or if there are severe curve differences between inspiration and expiration observed. Differences between inspiration and expiration are not only caused by a movable nasal entrance due to the measurement process, but also by different conditions of the fluid dynamics of the nose in a reversed air stream (Figure 6).

Histograms of the logarithmic effective resistance with marked classes as $20 \%$ percentiles of 10,033 measurements of untreated noses and 8,246 measurements after decongestion with $0.1 \%$ xylometazoline spray are depicted in Figure 7.

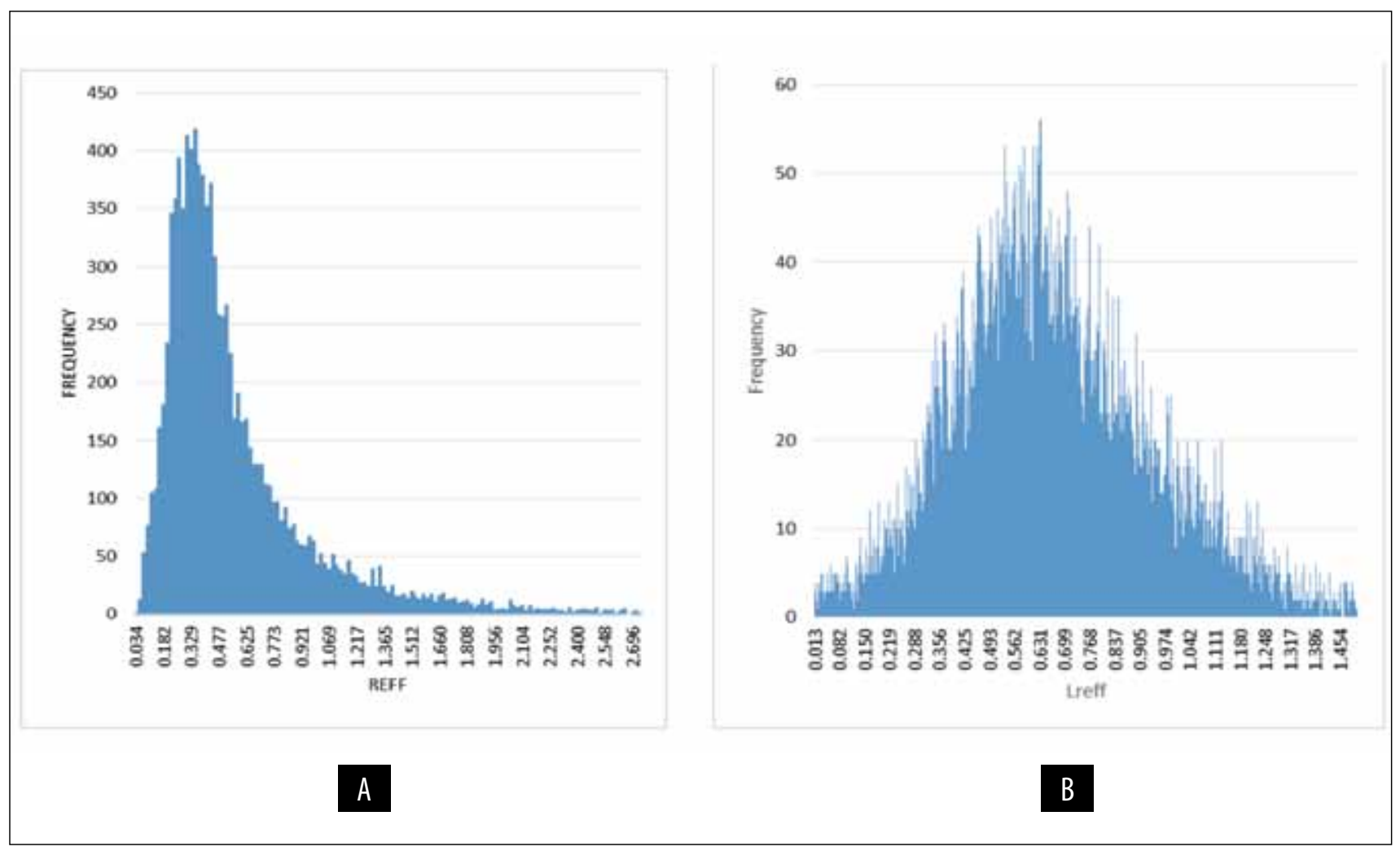

Figure 5 Statistical distribution of Reff (A) and LReff (B) in 10,033 cases 


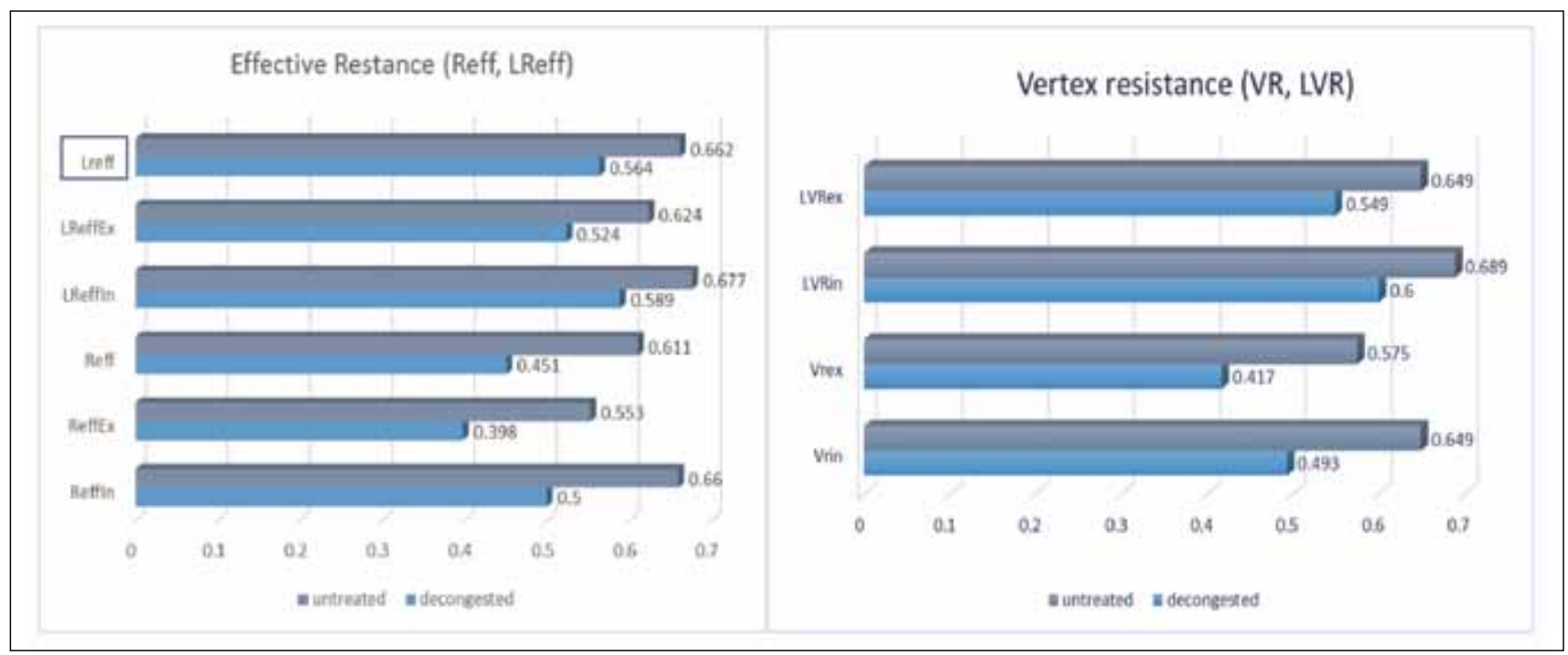

Figure 6 The variance of Effective and Vertex Resistance during inspiration and expiration

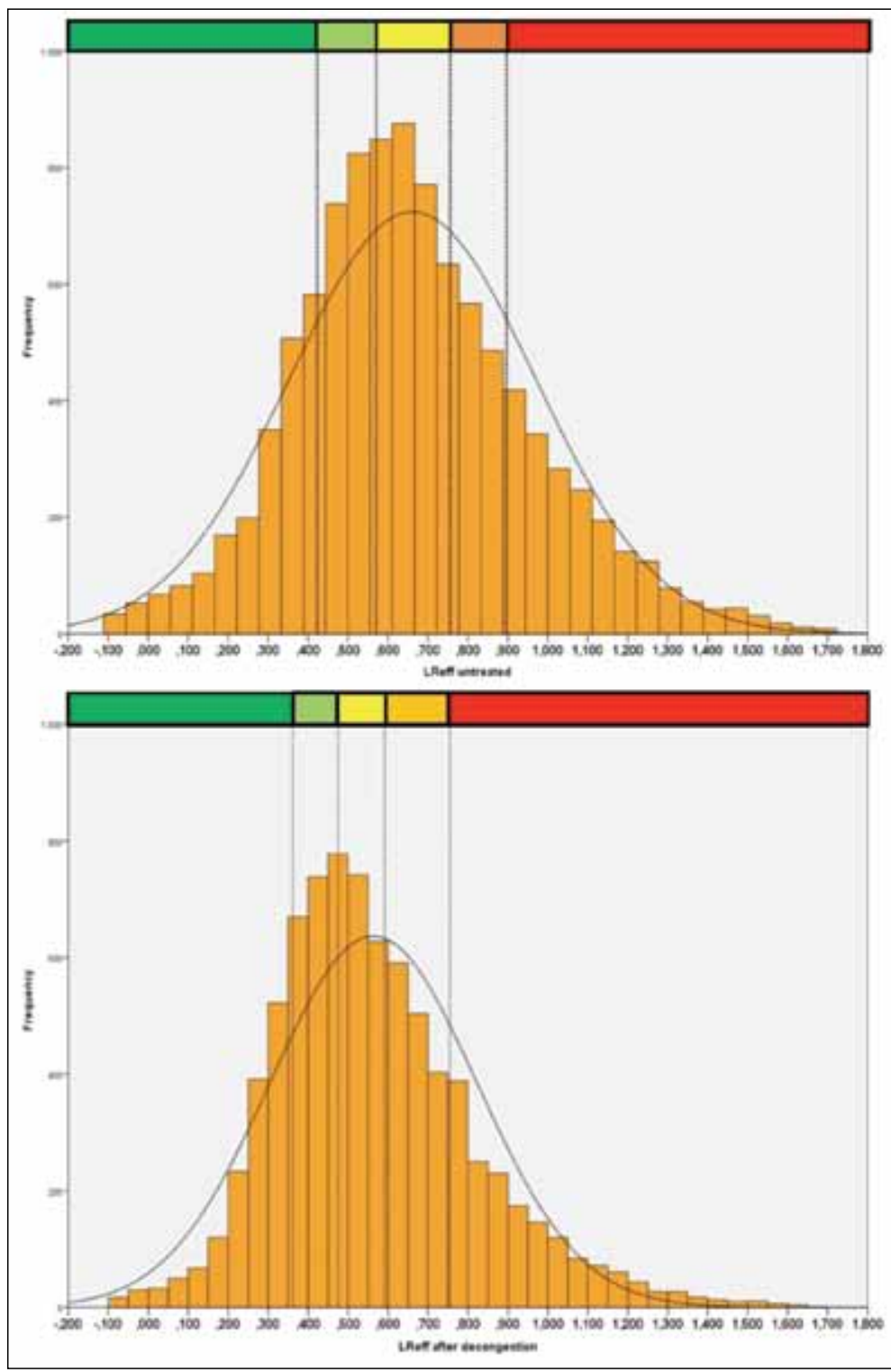

Figure 7 The logarithmic effective resistance with marked classes as 20\% percentiles 
Following the results above and including the results for unilateral measurements, a clinical classification for the nasal obstruction measured by 4-phaserhinomanometry results (Table 5 ).

A result of clinical interest may be also the comparison of the unilateral and total resistance (Reff) before and after decongestion (Table 6). That shows that the total resistance can be roughly estimated as one third lower than the unilateral resistance.

\section{DISCUSSIONS}

Similar investigations as in the pre-study of part 1 have been carried out already by Shelton and Eiser ${ }^{5}$, who did not find a statistic difference between posterior and anterior measurements of total resistance. The range of their resistance values was comparable to the classes of this publication and they confirmed that measurements by AAR are better reproducible than by APR. Jones, Lancer et al. ${ }^{6}$ found $16 \%$ higher values for measurements with posterior rhinomanometry; the small number of results is in agreement with the results above. In summary, by the prepara- tory study it is proved that the measurement of total nasal resistance by determination of the unilateral resistance in both sides and subsequent calculation has to be preferred, because posterior rhinomanometry generally is less reliable due to potential technical failures. It should be used in addition to anterior measurements, if the influence of the retropalatal space is of clinical interest.

The here-presented material allows for the first time a classification of the nasal obstruction by 4-phase - rhinomanometry in a statistically representative material using parameters which are obtained from technically correct measurements carried out with an identical technique in all investigating units and using physically and mathematically correct parameters with a correlation to the patients sensing of obstruction. The aim of this work is to define not only healthy persons by determination of so-called "normal values" or "reference intervals", but also to enable a surgeon to classify the degree of obstruction of a patient by comparing the individual result with a statistic representative population because of the possible consequences for the following necessary treatment and also for medico-legal reasons.

Table 5

Classification of nasal obstruction by unilateral and total nasal resistance (Logarithmic effective resistance (LReff))

\begin{tabular}{|c|c|c|c|c|c|}
\hline \multirow[b]{2}{*}{ Class } & & \multicolumn{2}{|c|}{ Unilateral Resistance } & \multicolumn{2}{|c|}{ Total Resistance } \\
\hline & & Untreated & after decongestion & Untreated & after decongestion \\
\hline 1 & 0-19\% & $<0.71$ & $<0.63$ & $<=0.42$ & $<=0.36$ \\
\hline 2 & $20-39 \%$ & $0.71-0.89$ & $0.63-0.78$ & $0.42-0.57$ & $0.36-0.47$ \\
\hline 3 & $40-59 \%$ & $0.89-1.08$ & $0.78-0.94$ & $0.57-0.70$ & $0.48-0.59$ \\
\hline 4 & $60-79 \%$ & $1.09-1.35$ & $0.94-1.18$ & $0.70-0.90$ & $0.59-0.76$ \\
\hline 5 & $80-100 \%$ & $>1.35$ & $>1.18$ & $>0.90$ & $>0.76$ \\
\hline
\end{tabular}

Table 6

Comparison of unilateral and total Logarithmic Effective Resistance (LReff)

Logarithmic Effective Resistance Reff

\begin{tabular}{ccccc}
\hline & Unilateral Resistance & Total Resistance \\
\hline & Untreated & after decongestion & Untreated & after decongestion \\
\hline mean & 1.03 & 0.92 & 0.66 & 0.56 \\
\hline & 20,069 & 16,495 & 10,033 & 8,246 \\
\hline
\end{tabular}


Moore and Eccles ${ }^{7}$ mentioned in their review the necessity to differentiate between the mucosal and skeletal resistance, because the mucosa is subject to the well-known influence of the circadian rhythm or nasal cycle, as well the influences of allergens and environment. Therefore, the values after decongestion tests are documented here too. The influence of growth and anthropometric parameters is a matter of our ongoing work, while the documentation of ethnic differences needs the international cooperation based on a common standard.

Because these parameters are still widely used, the values for the resistance at $150 \mathrm{~Pa}$ have to be compared with the results of the meta-analysis by Merkle et al. ${ }^{2}$ from 62 studies published from $1979-2013$. The study was carried out to obtain reference intervals for the "normal total airflow resistance" without considering the 4 phases of the breathing cycle. The only parameter analyzed was the resistance at a given differential pressure. Obviously, most of the publications used a reference pressure of $150 \mathrm{~Pa}$. Publications using a pressure of $75 \mathrm{~Pa}$ or the Broms method have been included. The published range for reference intervals of $0.12-0.38 \mathrm{~Pa} / \mathrm{cm}^{3} / \mathrm{s}$ with a mean of $0.25 \mathrm{~Pa} / \mathrm{cm}^{3} / \mathrm{s}$ for untreated noses, as well as the interval of $0.10-0.27 \mathrm{~Pa} / \mathrm{cm}^{3} / \mathrm{s}$ for decongested noses is comparable with the class $1-3$ in the classification of our clinical material. Only one of the studies, which was included in the meta-analysis (Kim et al.), presented statistically representative numbers of measurements, but, due to the ethnic differences between Asian and Caucasian noses, a comparison with the here-presented material is not possible.

The clear results of the here-presented analysis raise again the discussion about the parameters of the so-called "classic rhinomanometry". The following facts are strict arguments against the further use of these obsolete methods as taken over from historical graphical procedures used before the introduction of computerized rhinomanometry and later recommended by the ISOANA in 1984:

1. The measurement of the flow or the calculation of a resistance in $150 \mathrm{~Pa}$ is a measurement in an accelerating or decelerating phase of the breathing cycle. In "classic" rhinomanometry the point of its determination is not defined. Measurements in the ascending or descending, expiratory or inspiratory phases show significant differences. A simple look on time-related breathing curves shows these important differences clearly and instantly.

2. It was recommended and is popular to replace a measurement of the resistance in $150 \mathrm{~Pa}$ by a measurement in $75 \mathrm{~Pa}$ differential pressure, if the pressure level cannot be reached by the patient. As long as the flow values are compared, such a comparison is correct. To compare the resistances in $150 \mathrm{~Pa}$ and $75 \mathrm{~Pa}$ is physically nonsense, because the resistance depends on the different acceleration in the 4 breathing phases. The only one point with a linear relation between pressure and flow is the vertex of the curve in inspiration and expiration.

3. Not any measurement results of 1-point-measurements (including the "resistometry") are correlated with the sensing of obstruction, as it was shown in many publications dealing with this subject ${ }^{8}$ because their statistical distribution is not related to the logarithmic scale of sensing (Weber-Fechner's law). The systematic review about the highest level of evidence of a correlation between subjective and objective evaluation of the upper airway as given by André, Vuyk at al. ${ }^{8}$ in 2009 could not refer to 4-phase-rhinomanometry because at this time statistically relevant logarithmic data have not been available. Savovic et al. ${ }^{9}$ found recently a correlation between subjective obstruction and rhinomanometric results if the degree of obstruction increases - one more hint for the relation to the logarithmic scale of sensing.

In summary, misleading and unfounded statements as they have been recently given by Clement et al. ${ }^{10}$ and Wong and Eccles ${ }^{11}$ have to be rejected because they barricade the way of a meaningful development of nasal airway function tests. Under the auspices of Evidence Based Medicine it is not thinkable to use erroneous parameters in basic diagnostics.

The necessity to apply up to 5 parameters in $4-$ phase - rhinomanometry instead of 1 parameter in "classic" rhinomanometry" needs an interpretation. Measurable differences between the determined parameters in the same patient change the diagnostic algorithm: The rhinomanometric investigation is complete if the differences of inspiratory and expiratory values for effective and vertex resistance do not exceed the range of one class. In this case, LReff is the leading and sufficient parameter. If the effective resistance and the vertex resistance in inspiration are clearly higher as in expiration without loops appearing in the graph, the cause is very likely a rigid obstacle changing the shape of the nasal airstream after reversing the flow direction. In cases of differences between the inspiratory effective resistance and vertex resistance, the graphs show loops preferably in inspiration as signs of effective valve phenomena ${ }^{3}$. In this case, a second rhinomanometric test with forced inspiration leads to more information about the influence of the valve. 


\section{CONCLUSIONS}

The last decades of development of informatics and sensor techniques have shown that it is possible to develop measurement systems and software for easy practical use. The here-presented parameters for measuring the resistance of the nasal airway and the classification of the obstruction from a statistically representative material can be recommended for the daily practice in rhinology and rhinosurgery. An extension and storage of similar databases for public access, first with regard to nasal breathing in different ages and ethnic groups, should be a matter of an already started international cooperation.

Acknowledgments. Participating hospitals:

1. Parkklinik Weissensee, HNO-Abteilung (Prof. Dr. Hans Behrbohm), Berlin/Germany

2. Marienhospital Stuttgart, Abt. Plastische Chirurgie (Prof. Dr. W Gubisch), Stuttgart/Germany

3. Städtisches Klinikum Kassel, HNO-Klinik (Prof. Dr. U. Bockmühl), Kassel/Germany

4. Katholische Kliniken Ruhrhalbinsel, Abtl. Plastische Gesichtschirurgie (Dr. med Bombach), Essen/Germany

5. Achenbach-Krankenhaus Königs Wusterhausen, HNO-Abteilung (Dr. Zutelis). Königs Wusterhausen/Germany

Conflict of interest: The authors have no conflict of interest.

Contribution of authors: All authors have equally contributed to this work.

\section{REFERENCES}

1. Zicari A.M., Rugiano A., Ragusa G., Savastano V., Bertin S., Vittori T., Duse M. - The evaluation of adenoid hypertrophy and abstruction grading based on rhinomanometry after nasal decongestant in children. Eur Rev Med Pharmacol Sci., 2013;17(21):2962-2967.

2. Merkle J., Kohlhas L., Zadoyan G., Mösges R., Hellmich M. Rhinomanometric reference intervals for normal total airflow resistance. Rhinology, 2014;52(4):292-299. doi: 10.4193/Rhin13.220.

3. Vogt K., Jalowayski A.A., Althaus W., et al. - 4-Phase-Rhinomanometry (4PR) - basics and practice 2010. Rhinology, 2010;Suppl. 21:1-50.

4. Vogt K., Wernecke K.D., Behrbohm H., Gubisch W., Argale M. - Fourphase rhinomanometry: a multicentric retrospective analysis of 36,563 clinical measurements. Eur Arch Otorhinolaryngol., 2015;273(5):11851198. doi: 10.1007/s00405-015-3723-5. Epub 2015 Jul 22.

5. Shelton M., Eiser N. - Evaluation of active and posterior rhinomanometry in normal subjects. Clin Otolaryngol Allied Sci., 2007;17(2):178-182.

6. Jones A.S., Lancer J.M., Stevens J.C., Beckingham E. - Rhinomanometry: do the anterior and posterior methods give equivalent results? Clin Otolaryngol Allied Sci., 1987;12(2):109-114. doi: 10.1111/j.1365-2273.1987.tb00171.x

7. Moore M., Eccles R. - Normal nasal patency: problems in obtaining standard values for the surgeon. J Laryngol Otol., 2012;126(6):563-569. doi: 10.1017/S00222151120045X. Epub 2012 Apr 12.

8. Andre R.F., Vuyk H.D., Ahmed A., Graamans K., Nolst-Trenité G.J. Correlation between subjective and objective evaluation of the nasal airway.A systematic review of the highest level of incidence. Clin Otolraryngol., 2009;34(6):518-525. doi: 10.1111/j.1749-4486.2009.02042.x.

9. Savovic S., Smajic M., Molnar S., Jovancevic L., Buljcik-Cupic M., Kljajic V., Pilija V. - Correlation between subjective and objective nasal breathing assessments in examinees with nasal septum deformities. Vojnos anit Pregl., 2013;70(4):380-385. doi:10.2298/VSP1304380S.

10. Wong E.H.C., Eccles R. - Comparison of classic and 4-phase rhinomanometry methods, is there a difference? Rhinology, 2014;52(4):360-365. doi: 10.4193/Rhin

11. Clement P.A., Halewyck S., Gordts F., Michel O. - Critical evaluation of different objective techniques of nasal airway assessment: a critical review. Eur Arch Otorhinolaryngol., 2014;271(10):2617-2625. doi: 10.1007/s00405-013-2870-9. Epub 2014 Jan 20. 